\title{
The $N_{c}$ dependencies of baryon masses: Analysis with Lattice QCD and Effective Theory
}

\author{
A. Calle Cordón, ${ }^{1,2, *}$ T. DeGrand,$^{3, \dagger}$ and J. L. Goity ${ }^{1,4, \ddagger}$ \\ ${ }^{1}$ Thomas Jefferson National Accelerator Facility, Newport News, VA 23606, USA. \\ ${ }^{2}$ Departamento de Física, Universidad de Murcia, Murcia, E-30071 Murcia, Spain \\ ${ }^{3}$ Department of Physics, University of Colorado, Boulder, CO 80309, USA \\ ${ }^{4}$ Department of Physics, Hampton University, Hampton, VA 23668, USA.
}

\begin{abstract}
Baryon masses at varying values of $N_{c}$ and light quark masses are studied with Lattice QCD and the results are analyzed in a low energy effective theory based on a combined framework of the $1 / N_{c}$ and Heavy Baryon Chiral Perturbation Theory expansions. Lattice QCD results for $N_{c}=3,5$ and 7 obtained in quenched calculations, as well as results for unquenched calculations for $N_{c}=3$, are used for the analysis. The results are consistent with a previous analysis of $N_{c}=3$ Lattice QCD results, and in addition permit the determination of sub-leading in $1 / N_{c}$ effects in the spin-flavor singlet component of the baryon masses as well as in the hyperfine splittings.
\end{abstract}

PACS numbers: 11.15-Pg, 11.30-Rd, 12.39-Fe, 14.20-Dh

Keywords: Baryons, Lattice QCD, Large N, Chiral Perturbation Theory

*Electronic address: alvaro.calle@um.es

$\dagger$ Electronic address: thomas.degrand@colorado.edu

‡Electronic address: goity@jlab.org 


\section{Contents}

I. Introduction 2

II. Baryon masses in the combined $1 / N_{c}$ and chiral expansions 4

$\begin{array}{ll}\text { III. Lattice data sets } & 8\end{array}$

IV. The Effective Theory Analysis of LQCD Results 11

V. Conclusions 23

$\begin{array}{ll}\text { Acknowledgments } & 24\end{array}$

A. UV finite parts of the self-energy 26

$\begin{array}{ll}\text { References } & 27\end{array}$

\section{INTRODUCTION}

Lattice QCD (LQCD) calculations at varying number of colors $N_{c}$ provide the opportunity for understanding more quantitatively important aspects of hadronic physics which have been qualitatively described in terms of order of magnitude estimates based on $N_{c}$ power countings. Until recently, LQCD analyses at $N_{c}>3$ had been carried out only for pure gluon dynamics and for mesons, where first studies addressed confinement in $S U(2), S U(3)$ and $S U(4)$ Yang-Mills gauge theories [1], tests of the 't Hooft scaling in $g^{2} N_{c}=\lambda$, the relation between the QCD scale, $\Lambda_{Q C D}$ and the gauge coupling $g$ for different $N_{c}$ in [2-4], calculations of the glueball spectrum [5, 6], meson spectroscopy [7-9], masses and decay constants and their scalings with $N_{c}$ [10-12], the topological susceptibility [13]. For a review of these developments the reader can consult Ref. [14].

Recently, the extension to baryons, which is the focus of the present work, was performed by one of us, T. DeGrand in Ref. [12]. Baryon masses for $N_{c}=3,5$ and 7 were studied in the quenched approximation and at pion masses above $400 \mathrm{MeV}$. The results give a striking confirmation of the large $N_{c}$ QCD predictions for baryons, namely the $N_{c}$ scalings of baryon masses and of hyperfine (HF) mass splittings with $N_{c}$. That work also provides information 
on the quark mass dependence of baryon masses, which is exploited in the present work for the purpose of understanding the effective low energy theory based on the combined $1 / N_{c}$ expansion and Heavy Baryon Chiral Perturbation Theory (HBChPT) [15-20].

The importance of the $1 / N_{c}$ expansion in BChPT was pointed out long ago with the observation that the inclusion of the spin $\frac{3}{2}$ baryons leads to important cancellations in chiral loop corrections to axial currents [21, 22]. It was realized that such cancellations are a result of the constraints on the meson-baryon couplings in large $N_{c}[23,24]$, which gives rise to a contracted dynamical spin-flavor symmetry in baryons, which in particular requires the inclusion of the higher spin baryons, up to spin $N_{c} / 2$. This leads to the implementation of a baryon chiral Lagrangian following the strictures of the $1 / N_{c}$ expansion [15], a framework that has been further developed and applied in [16-20, 25]. The virtue of such an effective field theory $(\mathrm{EFT})$ is that it incorporates the dictates of the spin-flavor symmetry, emerging in the baryon sector of $\mathrm{QCD}$ at $N_{c} \rightarrow \infty$, into the chiral expansion and allowing for an unambiguous treatment of the spin- $\frac{3}{2}$ resonance as an explicit degree of freedom in the EFT.

In recent works [18-20], the EFT combining the $1 / N_{c}$ expansion and HBChPT was studied and applied to non-strange baryon masses and axial couplings (for a recent study of axial currents in $S U(3)$ see Ref. [25]). LQCD results at $N_{c}=3$ were used to determine lowenergy constants (LECs), and to test the low energy expansion. At small enough quark masses, where the low energy expansion holds, the $1 / N_{c}$ expansion is encoded in LEC's. Each operator in the chiral Lagrangian carries a LEC, which can be expanded in powers of $1 / N_{c}$. In order to determine the LECs, it is therefore necessary to have information at different values of $N_{c}$. This point is investigated with the LQCD results in the present work.

As has been already shown in Refs. [12, 26], LQCD baryon masses reproduce remarkably well the rotor spectrum predicted by the $1 / N_{c}$ expansion at large $N_{c}$. The most general mass formula is:

$$
m_{\mathbf{B}}\left(S, N_{c}, M_{\pi}\right)=N_{c} \mathbf{m}_{\mathbf{0}}\left(N_{c}, M_{\pi}\right)+\frac{\mathbf{C}_{\mathbf{H F}}\left(N_{c}, M_{\pi}\right)}{N_{c}} S(S+1)+\mathcal{O}\left(\frac{(S(S+1))^{2}}{N_{c}^{2}}\right),
$$

where $\mathbf{m}_{\mathbf{0}}$ and $\mathbf{C}_{\mathbf{H F}}$ start at $\mathcal{O}\left(N_{c}^{0} M_{\pi}^{0}\right)$, and can be determined with LQCD calculations and also studied with the EFT. Using the LQCD results [12], one can obtain an estimate:

$$
\begin{aligned}
\mathbf{C}_{\mathbf{H F}} & \sim \frac{4 N_{c}}{N_{c}\left(N_{c}+2\right)-3}\left(m_{\mathbf{B}}\left(S=\frac{N_{c}}{2}\right)-m_{\mathbf{B}}\left(S=\frac{1}{2}\right)\right) \\
\mathbf{m}_{\mathbf{0}} & \sim \frac{1}{N_{c}}\left(\bar{m}_{\mathbf{B}}-\left(\frac{1}{3}+\frac{1}{N_{c}}+\mathcal{O}\left(N_{c}^{-3}\right)\right)\left(m_{\mathbf{B}}\left(S=\frac{N_{c}}{2}\right)-m_{\mathbf{B}}\left(S=\frac{1}{2}\right)\right)\right),
\end{aligned}
$$


where $\bar{m}_{\mathbf{B}}$ is the average mass (or center of gravity) of the spin-flavor multiplet. A test of the rotor spectrum is provided by the ratio of the HF splittings between the $S=\frac{3}{2}$, $\frac{1}{2}$ states,

and the $S=\frac{N_{c}}{2},\left(\frac{N_{c}}{2}-1\right)$ states. The above formula gives 1.66 for $N_{c}=5$, while for all values of $M_{\pi}$ LQCD gives 1.5 to 1.6. For $N_{c}=7$ the formula gives 2.33 while LQCD gives 2 to 2.2 . These results are remarkably close to what is expected in the large $N_{c}$ limit.

There is one important point to mention concerning the definition of the $1 / N_{c}$ expansion. Since the $1 / N_{c}$ expansion compares different theories, there is an ambiguity in the very definition of the expansion. The ambiguity stems from the fact that the theories are defined at a renormalization scale, and that quantities run differently (by subleading corrections in $\left.1 / N_{c}\right)$ in the different theories. At the level of the fundamental parameters of QCD, the expansion can be defined by requiring that the 't Hooft coupling and the quark masses are the same, i.e. independent of $N_{c}$, at a chosen renormalization scale $\mu_{0}$. A different possibility is to choose hadronic quantities or observables which are $\mathcal{O}\left(N_{c}^{0}\right)$, such as $M_{\pi}$ and $F_{\pi} / \sqrt{N_{c}}$, and require them to be independent of $N_{c}$. What is precisely meant by the $1 / N_{c}$ expansion will therefore depend on the choice of definition scheme. In the present work a scheme defined at hadronic level and very similar to the one just mentioned will be utilized.

While performing the present study, it became apparent that the quenched LQCD data alone is not quite sufficient for establishing the robustness of the analysis, and thus it becomes necessary to include also results with dynamical quarks at $N_{c}=3$. This allows for a better control of the low quark mass domain. A careful discussion of the issues and viability of combining quenched and unquenched results will be presented.

This work is organized as follows: Section II gives a brief overview of the combined HBChPT and $1 / N_{c}$ expansion. Section III contains the LQCD results at varying $N_{c}$. Section IV contains the analysis, and Section $\mathrm{V}$ gives the conclusions. One Appendix gives explicit expressions for the finite parts of self energies and wave function renormalization factors needed in the calculations.

\section{BARYON MASSES IN THE COMBINED $1 / N_{c}$ AND CHIRAL EXPANSIONS}

Baryon observables have peculiar scalings in $N_{c}$ : their masses scale as $N_{c}$, while the mass differences between states with spins $\mathcal{O}\left(N_{c}^{0}\right)$ scale as $1 / N_{c}$. The pion couplings to baryons scale as $\sqrt{N_{c}}$, which has profound consequences for the baryon chiral expansion. In fact, 
these peculiar scalings give rise in the large $N_{c}$ limit to a dynamical spin-flavor symmetry for baryons. For $N_{f}$ degenerate flavors, that dynamical symmetry is a contracted $S U\left(2 N_{f}\right)$ symmetry $[23,24,27,28]$, which is broken by the fact that $N_{c}$ is finite. For $N_{c}$ sufficiently large those effects can be expanded in powers of $1 / N_{c}$. Although there is no rigorous proof, there are phenomenological indications as well as the LQCD results analyzed here which suggest that an expansion, modulo the subtleties discussed below, can be implemented for $N_{c}$ down to the real world's value $N_{c}=3$.

When the $1 / N_{c}$ expansion is combined with the low energy expansion, it is found that the chiral and $1 / N_{c}$ expansions do not commute due to the presence of the ratio $M_{\pi} /\left(m_{\Delta}-\right.$ $\left.m_{N}\right)=\mathcal{O}\left(p N_{c}\right)$ in the non-analytic pieces of chiral loop contributions [29]. In the meson sector, the expansions do commute with each other [30] except for the effects of the $\eta-\eta^{\prime}$ mixing, which involve the product $N_{c}\left(m_{s}-\hat{m}\right)$. ( $\hat{m}$ is the average nonstrange quark mass.) In particular, the non-commutativity of the expansions requires that the two power countings ought to be linked for a definite EFT to be defined. A linking which seems to be the most adequate for the real world baryons is the $\xi$-expansion [18], where the countings are linked according to $\mathcal{O}\left(1 / N_{c}\right)=\mathcal{O}(p)=\mathcal{O}(\xi)$. The chosen power counting scheme determines the terms in the effective chiral Lagrangian at each given order of the expansion. In the $\xi$ expansion, the Lagrangian for the combined BChPT and $1 / N_{c}$ expansions to order $\xi$, following Refs. [15, 18], reads:

$$
\mathcal{L}_{\mathbf{B}}^{(1)}=\mathbf{B}^{\dagger}\left(i D_{0}+\stackrel{\circ}{g}_{A} u^{i a} G^{i a}-\frac{m_{2}}{N_{c}}-\frac{C_{H F}}{N_{c}} \vec{S}^{2}-\frac{c_{1}}{2} N_{c} \chi_{+}\right) \mathbf{B},
$$

where only the case of two flavors is considered. $\mathbf{B}$ is the symmetric spin-flavor baryon multiplet with states $S=I=\frac{1}{2}, \cdots, \frac{N_{c}}{2} ; G^{i a}$ are the spin-flavor generators of $S U(4)$ with $i$ spatial indices and $a$ isospin indices and matrix elements $\mathcal{O}\left(N_{c}\right)$. The pions reside in the chiral covariant derivative $D_{0}$ and in $u^{i a}=\frac{1}{2} \operatorname{Tr}\left(\tau^{a} u^{i}\right)$, where $u^{i}=-\frac{1}{F_{\pi}} \partial^{i} \vec{\pi} \cdot \vec{\tau}+\cdots$, and the quark masses reside in $\chi_{+}=2 M_{\pi}^{2}+\cdots$. The LECs $m_{2}, \stackrel{\circ}{g}_{A}, C_{H F}$, and $c_{1}$ are $\mathcal{O}\left(N_{c}^{0}\right)$. As defined here, the axial coupling $\stackrel{\circ}{g}_{A}$ is related to the one of the nucleon at $N_{c}=3$ by $\stackrel{\circ}{g}_{A}=\frac{6}{5} g_{A}$, where $g_{A}=1.27$ is the well known nucleon axial coupling.

The baryon masses to one loop are as follows [18]:

$$
\begin{aligned}
m_{\mathbf{B}}(S) & =N_{c} m_{0}\left(N_{c}\right)+c_{1} N_{c} M_{\pi}^{2}+\frac{C_{H F}}{N_{c}} S(S+1) \\
& +\left\langle\mathbf{B}\left|\frac{\left.\left(\delta \Sigma_{(1-\text { looo })}^{U V}+\delta \Sigma^{C T}\right)\right|_{\mathfrak{p}^{0}=0}}{1-\delta Z_{(1-\text { loop })}^{U V}-\delta Z^{C T}}\right| \mathbf{B}\right\rangle,
\end{aligned}
$$


where $S$ is the spin of the baryon, and $m_{0}\left(N_{c}\right)=m_{0}+\frac{m_{1}}{N_{c}}+\cdots$. The rest mass which is removed by the heavy baryon expansion is the term $m_{0}+\frac{m_{1}}{N_{c}}$. The one-loop finite pieces are evaluated in dimensional regularization and MS scheme at a renormalization scale $\mu$, whose dependence is canceled by that of the counter-terms (indicated with the label $C T$ ). $\mathfrak{p}^{0}$ is the residual baryon energy, and as usual, the correction to the wave function renormalization factor is defined by $\delta Z=\partial \delta \Sigma / \partial \mathfrak{p}^{0}$. The last term on the RHS of Eq. (4) contains the contributions $\mathcal{O}\left(\xi^{2}\right)$ and $\mathcal{O}\left(\xi^{3}\right)$. All the details on the derivation of Eq. (4) are found in Ref. [18].

The UV divergences are given by [18]:

$$
\begin{aligned}
\left.\delta \Sigma(S)^{U V}\right|_{\mathfrak{p}^{0}=0} & =\frac{\stackrel{\circ}{g}_{A}^{2} C_{H F}}{16 \pi^{2} \stackrel{\circ}{F}_{0}^{2}}\left(1+\frac{4}{N_{c}}\right)\left(-\frac{9}{8} M_{\pi}^{2}+\frac{C_{H F}^{2}}{N_{c}^{2}}(3+5 S(S+1))\right) \lambda_{\epsilon} \\
\delta Z(S)^{U V} & =\frac{3 \stackrel{\circ}{2}_{A}^{2}}{512 \pi^{2} \stackrel{\circ}{F}_{0}^{2}}\left(N_{c}+4\right)\left(3 M_{\pi}^{2}-\frac{8 C_{H F}^{2}}{N_{c}^{2}}(3+2 S(S+1))\right) \lambda_{\epsilon}
\end{aligned}
$$

where $\stackrel{\circ}{F}_{0} \equiv \stackrel{\circ}{F}_{\pi} \sqrt{3 / N_{c}}$ with $\stackrel{\circ}{F}_{\pi}$ the pion decay constant in the chiral limit, $\lambda_{\epsilon}=\frac{1}{\epsilon}-\gamma+\log 4 \pi$, and the counter-terms necessary for the renormalization of the self-energy at $\mathcal{O}\left(\xi^{3}\right)$ read as follows [18]:

$$
\begin{aligned}
\delta \Sigma^{C T}\left(\mathfrak{p}^{0}=0\right)(S) & =\mu_{1} M_{\pi}^{2}+\left(\frac{\mu_{2}}{N_{c}} M_{\pi}^{2}+\frac{C_{H F 1}}{N_{c}^{2}}\right) S(S+1)+\frac{m_{3}}{N_{c}^{2}}+\frac{m_{4}}{N_{c}^{3}}+\frac{C_{H F 2}}{N_{c}^{3}}(S(S+1))^{2} \\
\delta Z^{C T}(S) & =z_{1} N_{c} M_{\pi}^{2}+\frac{w_{1}}{N_{c}}+\frac{w_{2}}{N_{c}} S(S+1)+\mathcal{O}\left(\xi^{2}\right) .
\end{aligned}
$$

To one loop, $\delta \Sigma^{C T}$ has terms $\mathcal{O}\left(\xi^{2}\right)$ and $\mathcal{O}\left(\xi^{3}\right)$, and $\delta Z^{C T}$ has terms $\mathcal{O}(\xi)$ and $\mathcal{O}\left(\xi^{2}\right)$. In the result for the masses, Eq. (4), only the terms $\mathcal{O}(\xi)$ in $\delta Z^{C T}$ are relevant. The LECs depend on $\mu$ in order to render the result for the masses $\mu$ independent. Comparison with Eq. (5) shows that several of the counter-terms are finite, as they are not required to cancel the UV divergences shown in Eq. (5). The masses so calculated are accurate to $\mathcal{O}\left(\xi^{3}\right)$. For completeness, the explicit expressions of the finite pieces of the self-energy are given in the Appendix.

In the large $N_{c}$ limit, the spin-flavor singlet one-loop contributions to the baryon selfenergy show UV finite terms $\mathcal{O}\left(M_{\pi}^{3} N_{c}\right)$, and both UV finite and divergent terms $\mathcal{O}\left(\mathfrak{p}^{0} M_{\pi}^{2} N_{c}\right)$ which affect the wave function renormalization factor [18] . This implies the breakdown of the low energy expansion for the spin-flavor singlet components of the self-energy in the strict limit $N_{c} \rightarrow \infty$. On the other hand, the spin-flavor non-singlet components of the selfenergy, i. e., the HF splittings, are suppressed by at least one power of $1 / N_{c}$. Cancellations 
of contributions that violate that power behavior lead to an improved convergence of the low energy expansion of the HF splittings. This is shown in the analysis below, where HF splittings are consistently described in a larger range of $M_{\pi}$ than the spin-flavor singlet masses.

It is important to emphasize that the effects of quenching do not qualitatively change the above arguments. In fact, for each operator which appears in the effective Lagrangian, the leading LEC of $\mathcal{O}\left(N_{c}^{0}\right)$ should be the same in quenched and unquenched cases. At the quark level one can visualize the leading contributions in $N_{c}$ to masses and wave function renormalization factors by the diagram shown in Fig. 1, which corresponds to the quenched case.

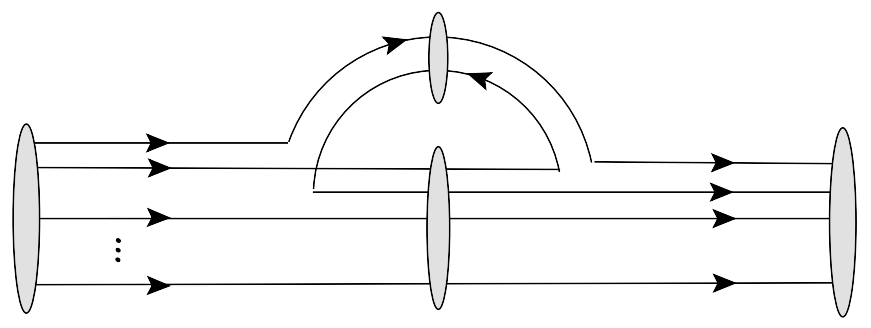

FIG. 1: Dominant contribution by pion-loop to baryon self-energy $\left(\mathcal{O}\left(N_{c}\right)\right)$. Diagrams with a quark loop are suppressed by a factor $1 / N_{c}$. The blobs indicate hadrons.

Quantitatively, quenching does have an effect, which was first described by Labrenz and Sharpe [31, 32]: In "real" QCD with dynamical sea quarks, the generic baryon mass is

$$
M_{B}=M_{0}+c_{2} M_{\pi}^{2}+c_{3} M_{\pi}^{3}+C_{4 L} M_{\pi}^{4} \log M_{\pi}+c_{4} M_{\pi}^{4}+\ldots
$$

(the $c_{3}$ term arises from the one loop pion exchange graph). In quenched QCD, the mass is

$$
M_{B}^{q}=M_{0}^{q}+\left[\delta c_{1}^{q} M_{\pi}+\delta c_{2 L}^{q} M_{\pi}^{2} \log M_{\pi}\right]+c_{3}^{q} M_{\pi}^{3}+C_{4 L}^{q} M_{\pi}^{4} \log M_{\pi}+c_{4}^{q} M_{\pi}^{4}+\ldots
$$

The superscript $q$ reminds us that the quenched coefficients need have no connection to the unquenched ones. The extra terms are the quenching artifacts. $\delta=m_{0}^{2} /\left(48 \pi^{2} f^{2}\right)$ and $m_{0}$ is a number associated with the annihilation graph $q \bar{q} \rightarrow q \bar{q}$. The extra terms come when the baryon emits and absorbs $q \bar{q}$ pairs which would like to iterate into the eta (and then not 
be present in a chiral expansion), but because it is quenched approximation, the iteration terminates. It gives a double pole chiral loop. For the nucleon, $c_{1}^{q}=3 \pi / 2(3 F-D)^{2}$. As we are interested in large $N_{c}$, a later paper by Chow and Rey [33] makes a useful point: the $m_{0}^{2}$ parameter in $\delta$ is also $O\left(1 / N_{c}\right)$, so $\delta$ scales like $1 / N_{c}^{2}$. This means that both of these quenching artifacts, the extra term in the expansion and the difference between quenched and unquenched LEC's, are nonleading in $1 / N_{c}$ compared to the usual chiral factors. They would appear as one of many corrections to the leading terms in the chiral expansion.

One could opt to restrict the analysis of this work only to the quenched data sets of Ref. [12]. However, the spectroscopies of the $S U(3)$ quenched data set and of the unquenched $S U(3)$ data sets, conveniently scaled by using the chiral limit $F_{\pi}$ to set the scale, are found to be consistent within uncertainties and so phenomenologically can be combined. A test using only quenched or unquenched data will also be done, in order to avoid combining quenched and unquenched data sets into a single fit.

\section{LATTICE DATA SETS}

The lattice data for $N_{c}=5$ and 7 is based largely on that of Ref. [12]. Readers should refer there, and to a later paper focused on flavor $S U(3)$ symmetry, [26], for more details. Here is a brief summary: Simulations use the usual Wilson plaquette gauge action, with clover fermions with normalized hypercubic smeared links as their gauge connections[34]. The clover coefficient is fixed at its tree level value, $c_{S W}=1$. The code is a version of the publicly available package of the MILC collaboration [35].

All simulations are performed in quenched approximation. The simulation volumes were all $16^{3} \times 32$ sites. The bare gauge couplings were (roughly) matched so that pure gauge observables were the same on all three $N_{c}$ 's, so as to match discretization and finite volume effects. The observable chosen to do the comparison was the shorter version of the Sommer parameter [36] $r_{1}$, defined in terms of the force $F(r)$ between static quarks, $r^{2} F(r)=-1.0$ at $r=r_{1}$. The real-world value is $r_{1}=0.31 \mathrm{fm}$ [37], and with it the common lattice spacing is about $0.08 \mathrm{fm}$.

Simulation parameters are reported in Table I. The masses of the pseudoscalar and vector mesons, the pseudoscalar decay constant, and the baryon masses are shown in Tables III-IV, and the HF splittings are shown in Tables V through VII. 


\begin{tabular}{cccc}
\hline & $S U(3)$ & $S U(5)$ & $S U(7)$ \\
\hline$\beta$ & 6.0175 & 17.5 & 34.9 \\
configurations & 80 & 120 & 160 \\
$r_{1} / a$ & $3.90(3)$ & $3.77(3)$ & $3.91(2)$ \\
\hline
\end{tabular}

TABLE I: Parameters characterizing the simulations.

\begin{tabular}{ccccccc}
\hline$\kappa$ & $a m_{q}$ & $a M_{\pi}$ & $a F_{\pi}$ & $a m_{V}$ & $a m_{B}\left(S=\frac{3}{2}\right)$ & $a m_{B}\left(S=\frac{1}{2}\right)$ \\
\hline 0.1220 & 0.148 & $0.598(1)$ & $0.077(1)$ & $0.676(2)$ & $1.089(6)$ & $1.035(5)$ \\
0.1230 & 0.119 & $0.527(1)$ & $0.072(1)$ & $0.619(3)$ & $1.007(7)$ & $0.942(6)$ \\
0.1240 & 0.089 & $0.449(2)$ & $0.067(1)$ & $0.554(4)$ & $0.926(10)$ & $0.845(8)$ \\
0.1245 & 0.074 & $0.407(2)$ & $0.064(1)$ & $0.522(5)$ & $0.886(11)$ & $0.795(8)$ \\
0.1250 & 0.059 & $0.361(2)$ & $0.062(1)$ & $0.490(6)$ & $0.846(13)$ & $0.744(10)$ \\
0.1253 & 0.050 & $0.331(2)$ & $0.059(1)$ & $0.470(7)$ & $0.821(15)$ & $0.711(10)$ \\
0.1257 & 0.038 & $0.288(2)$ & $0.056(1)$ & $0.449(8)$ & $0.786(18)$ & $0.663(12)$ \\
0.1260 & 0.029 & $0.253(2)$ & $0.054(1)$ & $0.428(11)$ & $0.757(19)$ & $0.621(13)$ \\
0.1261 & 0.026 & $0.240(2)$ & $0.054(1)$ & $0.421(12)$ & $0.747(20)$ & $0.606(13)$ \\
0.1262 & 0.022 & $0.225(3)$ & $0.053(1)$ & $0.445(9)$ & $0.736(20)$ & $0.589(14)$ \\
0.1265 & 0.013 & $0.177(3)$ & $0.052(1)$ & $0.428(12)$ & $0.697(24)$ & $0.527(17)$ \\
0.1266 & 0.010 & $0.156(4)$ & $0.051(1)$ & $0.421(14)$ & $0.677(29)$ & $0.495(22)$ \\
0.1267 & 0.006 & $0.133(6)$ & $0.051(1)$ & $0.432(13)$ & $0.643(43)$ & $0.449(40)$ \\
\hline
\end{tabular}

TABLE II: Masses in lattice units for the $S U(3)$ data sets. From left to right, the entries are the hopping parameter $\kappa$, the Axial Ward Identity quark mass, the pseudoscalar mass, the pseudoscalar decay constant, the vector meson mass, and the baryons, labeled by their spin $S$.

The data are extended from that of Ref. [12] in two ways. First, the $N_{c}=7, S=\frac{1}{2}$ baryon was added to the set of measured states. As described in Ref. [12], lower $S$ states contain many more contractions of creation and annihilation operators into propagators. The new baryon's propagator has about 1.5 million determinants needed to be evaluated, per site. Second, the spectroscopy has been extended for all $N_{c}$ 's to lower quark mass. Comparisons of large- $N_{c}$ predictions do not necessary require small quark mass, but of course chiral extrapolations need the lightest possible quark masses. The quark mass was 


\begin{tabular}{|c|c|c|c|c|c|c|c|}
\hline$\kappa$ & $a m_{q}$ & $a M_{\pi}$ & $a F_{\pi}$ & $a m_{V}$ & $a m_{B}\left(S=\frac{5}{2}\right)$ & $a m_{B}\left(S=\frac{3}{2}\right)$ & $a m_{B}\left(S=\frac{1}{2}\right)$ \\
\hline 0.1240 & 0.127 & $0.565(1)$ & $0.097(1)$ & $0.655(1)$ & $1.866(7)$ & $1.817(6)$ & $1.787(6)$ \\
\hline 0.1250 & 0.098 & $0.488(1)$ & $0.090(1)$ & $0.593(1)$ & $1.711(7)$ & $1.652(6)$ & $1.617(6)$ \\
\hline 0.1260 & 0.070 & $0.403(1)$ & $0.083(1)$ & $0.532(2)$ & $1.562(7)$ & $1.490(7)$ & $1.448(6)$ \\
\hline 0.1265 & 0.055 & $0.356(1)$ & $0.079(1)$ & $0.500(2)$ & $1.482(8)$ & $1.402(7)$ & $1.355(6)$ \\
\hline 0.1270 & 0.041 & $0.302(2)$ & $0.073(1)$ & $0.469(3)$ & $1.419(11)$ & $1.324(8)$ & $1.270(7)$ \\
\hline 0.1275 & 0.026 & $0.240(2)$ & $0.069(1)$ & $0.440(4)$ & $1.361(10)$ & $1.250(13)$ & $1.184(10)$ \\
\hline 0.1278 & 0.017 & $0.193(3)$ & $0.065(1)$ & $0.424(5)$ & $1.284(12)$ & $1.163(15)$ & $1.085(13)$ \\
\hline 0.1280 & 0.009 & $0.155(7)$ & $0.063(1)$ & $0.413(7)$ & $1.247(20)$ & $1.105(36)$ & $1.006(31)$ \\
\hline
\end{tabular}

TABLE III: Masses in lattice units for the $S U(5)$ data sets.

\begin{tabular}{|c|c|c|c|c|c|c|c|c|}
\hline$\kappa$ & $a m_{q}$ & $a M_{\pi}$ & $a F_{\pi}$ & $a m_{V}$ & $a m_{B}\left(S=\frac{7}{2}\right)$ & $a m_{B}\left(S=\frac{5}{2}\right)$ & $a m_{B}\left(S=\frac{3}{2}\right)$ & $a m_{B}\left(S=\frac{1}{2}\right)$ \\
\hline 0.1260 & 0.115 & $0.565(1)$ & $0.115(1)$ & $0.663(1)$ & $2.668(11)$ & $2.625(10)$ & $2.595(10)$ & $2.577(10)$ \\
\hline 0.1270 & 0.088 & $0.488(1)$ & $0.107(1)$ & $0.603(1)$ & $2.471(13)$ & $2.420(12)$ & $2.383(11)$ & $2.361(11)$ \\
\hline 0.1280 & 0.062 & $0.401(1)$ & $0.097(1)$ & $0.537(2)$ & $2.273(18)$ & $2.213(13)$ & $2.166(13)$ & $2.139(12)$ \\
\hline 0.1290 & 0.036 & $0.299(1)$ & $0.086(1)$ & $0.471(3)$ & $2.075(28)$ & $1.998(17)$ & $1.938(17)$ & $1.904(18)$ \\
\hline 0.1295 & 0.022 & $0.235(2)$ & $0.081(1)$ & $0.438(4)$ & $1.967(27)$ & $1.891(20)$ & $1.818(21)$ & $1.777(22)$ \\
\hline 0.1297 & 0.017 & $0.205(2)$ & $0.078(1)$ & $0.426(5)$ & $1.924(34)$ & $1.843(26)$ & $1.773(26)$ & \\
\hline 0.1298 & 0.013 & $0.178(3)$ & $0.076(1)$ & $0.427(4)$ & $1.890(45)$ & $1.833(40)$ & $1.735(38)$ & \\
\hline
\end{tabular}

TABLE IV: Masses in lattice units for the $S U(7)$ data sets.

lowered until at very small quark mass "exceptional configurations" are encountered. These are gauge configurations on which the Dirac operator has eigenvalues close to the value zero, so it becomes ill-conditioned, and hence non-invertible. This situation is well-known from quenched studies in $N_{c}=3$. There, it is cured by doing simulations with dynamical fermions, since the zero modes mean that the fermion determinant is zero and these configurations never appear in the Markov chain.

In any lattice calculation, the values of two observables computed on the same set of lattice configurations are highly correlated. In the case of mass differences, this means that the uncertainty in $m(S)-m\left(S^{\prime}\right)$ is smaller than what a naive combination of the individual 


\begin{tabular}{cc}
\hline$\kappa$ & $\Delta m_{B}\left(\frac{3}{2}, \frac{1}{2}\right)$ \\
\hline 0.1220 & $0.054(3)$ \\
0.1230 & $0.065(4)$ \\
0.1240 & $0.080(6)$ \\
0.1245 & $0.091(7)$ \\
0.1250 & $0.102(8)$ \\
0.1253 & $0.110(10)$ \\
0.1257 & $0.123(12)$ \\
0.1260 & $0.136(14)$ \\
0.1261 & $0.141(14)$ \\
0.1262 & $0.147(15)$ \\
0.1265 & $0.170(21)$ \\
0.1266 & $0.181(28)$ \\
0.1267 & $0.195(49)$ \\
\hline
\end{tabular}

TABLE V: HF split-

\begin{tabular}{cccc}
\hline$\kappa$ & $\Delta m_{B}\left(\frac{5}{2}, \frac{3}{2}\right)$ & $\Delta m_{B}\left(\frac{5}{2}, \frac{1}{2}\right)$ & $\Delta m_{B}\left(\frac{3}{2}, \frac{1}{2}\right)$ \\
\hline 0.1240 & $0.050(2)$ & $0.080(2)$ & $0.030(1)$ \\
0.1250 & $0.059(2)$ & $0.094(3)$ & $0.035(1)$ \\
0.1260 & $0.071(2)$ & $0.114(4)$ & $0.043(2)$ \\
0.1265 & $0.080(3)$ & $0.127(5)$ & $0.047(2)$ \\
0.1270 & $0.096(7)$ & $0.150(9)$ & $0.054(4)$ \\
0.1275 & $0.112(11)$ & $0.177(10)$ & $0.066(12)$ \\
0.1278 & $0.121(13)$ & $0.200(13)$ & $0.079(11)$ \\
0.1280 & $0.142(34)$ & $0.241(37)$ & $0.099(45)$ \\
\hline
\end{tabular}

TABLE VI: HF splittings for $N_{c}=5$

\begin{tabular}{ccccc}
\hline$\kappa$ & $\Delta m_{B}\left(\frac{7}{2}, \frac{5}{2}\right)$ & $\Delta m_{B}\left(\frac{7}{2}, \frac{3}{2}\right)$ & $\Delta m_{B}\left(\frac{5}{2}, \frac{3}{2}\right)$ & $\Delta m_{B}\left(\frac{5}{2}, \frac{1}{2}\right)$ \\
\hline 0.1260 & $0.042(4)$ & $0.072(6)$ & $0.030(2)$ & $0.019(1)$ \\
0.1270 & $0.051(4)$ & $0.088(7)$ & $0.037(3)$ & $0.022(1)$ \\
0.1280 & $0.060(13)$ & $0.106(13)$ & $0.046(5)$ & $0.027(2)$ \\
0.1290 & $0.077(22)$ & $0.136(24)$ & $0.059(8)$ & $0.035(4)$ \\
0.1295 & $0.076(21)$ & $0.149(20)$ & $0.073(14)$ & $0.041(14)$ \\
0.1297 & $0.081(22)$ & $0.151(27)$ & $0.071(14)$ & \\
0.1298 & $0.057(42)$ & $0.156(45)$ & $0.098(36)$ & \\
\hline
\end{tabular}

TABLE VII: HF splittings for $N_{c}=7$.

uncertainties in $m(S)$ and $m\left(S^{\prime}\right)$ would indicate. The baryon masses and the mass differences were simultaneously computed by performing a single-elimination jackknife analysis of the appropriate correlators. This ensures that the difference of masses (as recorded, for example, in Table II) is equal to the mass differences (as recorded in Table V).

\section{THE EFFECTIVE THEORY ANALYSIS OF LQCD RESULTS}

This section presents the analysis of the LQCD results of the previous section in the framework of the combined HBChPT and $1 / N_{c}$ expansions with the $\xi$ power counting [18]. This analysis should be considered as a first attempt at determining the $N_{c}$ dependencies 
of baryon masses in that expansion from the first LQCD results for baryons at varying $N_{c}$. The main limitation of the present analysis is the current range of quark masses in the simulations, which corresponds to $350 \mathrm{MeV} \leq M_{\pi} \leq 1400 \mathrm{MeV}$. Due to that limitation and in order to have more information at lower values of $M_{\pi}$, inputs from other LQCD calculations at $N_{c}=3$ will be included [38-40]. This, however, introduces a different limitation, which is the matching between full QCD results at $N_{c}=3$ and the results of the previous section performed in the quenched approximation. For quark masses in the domain of Ref [12], quenching effects are expected to be relatively small. It is obvious that for a more accurate analysis the simulations at varying $N_{c}$ must be carried out at lower quark masses, where at the same time dynamical quarks will be needed, which is perhaps at this time a very challenging task. Nevertheless, at the present level of accuracies such a combination seems to be justified as it is shown by the following discussion.

In general, the effects of quenching on hadron observables are suppressed by a factor $1 / N_{c}$, as it follows from 't Hooft's $N_{c}$ power counting (exceptions exist, such as the topological susceptibility which is $\mathcal{O}\left(N_{c}^{0}\right)$ in the quenched approximation and vanishes in the chiral limit in full QCD [41]). In baryons, quenching affects baryon masses at $\mathcal{O}\left(N_{c}^{0}\right)$, but this effect respects spin-flavor symmetry, while in the HF splittings it is an effect $\mathcal{O}\left(1 / N_{c}^{2}\right)$. Thus, to be rigorously consistent with sub-leading orders in the $1 / N_{c}$ expansion, the LQCD calculations will need to be in full QCD, which is of course increasingly expensive with $N_{c}$. Nonetheless, QCD in the quenched approximation itself admits a $1 / N_{c}$ expansion, and it is therefore of interest in its own right.

The issue of combining quenched and unquenched data is now analyzed. The combination of $N_{c}=3$ quenched and unquenched results is found to work remarkably well when quantities are considered in units of the corresponding $\stackrel{\circ}{F}_{0}$, defined as the chiral limit value of $F_{0} \equiv$ $F_{\pi} \sqrt{3 / N_{c}}$. In particular, for the quantities of relevance to the present analysis, namely $F_{0} / \stackrel{\circ}{F}_{0}$ vs $M_{\pi} / \stackrel{\circ}{F}_{0}$ and $M_{B} / \stackrel{\circ}{F}_{0}$ vs $M_{\pi} / \stackrel{\circ}{F}_{0}$, there is agreement between the quenched and the unquenched results within the current LQCD errors, as is shown below.

First the case of pions is discussed at the level of the two quantities of relevance for this work, namely $M_{\pi}$ and $F_{\pi}$. Since $\stackrel{\circ}{F}_{0}$ is crucial in the present analysis, a detailed discussion is given of its determination and consistency for the different LQCD calculations being 

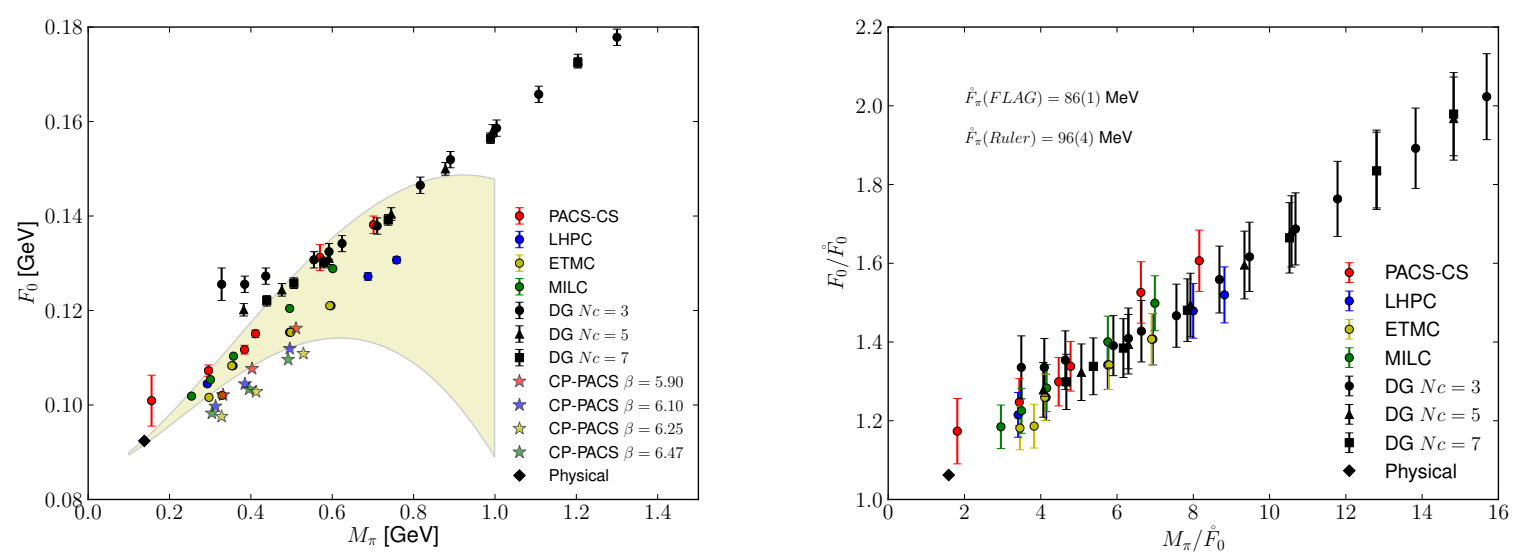

FIG. 2: Left panel: $F_{0} \equiv F_{\pi} \sqrt{3 / N_{c}}$ vs $M_{\pi}$ in physical units for the quenched results from CPPACS [42] and $N_{c}=3,5,7$ of Ref. [12], and the unquenched results from PACS-CS [38], LHPC [39], ETMC [40] and MILC [43] collaborations. The diamond represents the physical point. The yellow band represents $\mathcal{O}\left(p^{4}\right)$ ChPT [44] with the recent FLAG's determination of the LECs $\bar{\ell}_{4}$ and $\stackrel{\circ}{F}_{\pi}$ from Eqs. (10) and (9) (see Ref. [45]). Right panel: $F_{0} / \stackrel{\circ}{F}_{0}$ vs $M_{\pi} / \stackrel{\circ}{F}_{0} . \stackrel{\circ}{F}_{0}$ is determined from the FLAG's averaged value in the case of unquenched LQCD results and the linear extrapolation value in the case of the $N_{c}=3,5,7$ results of Ref. [12].

considered. In Fig. 2, $F_{0}$ vs. $M_{\pi}$ is displayed for several LQCD calculations. The left panel displays results from different LQCD collaborations in quenched (DeGrand and CPPACS) and full QCD (PACS-CS, LHPC, ETMC and MILC). Results are displayed using the quoted lattice spacings provided by the different collaborations, namely, $a \simeq 0.08 \mathrm{fm}$ (DeGrand) [12], $a \simeq 0.09 \mathrm{fm}$ (PACS-CS) [38], $a \simeq 0.124 \mathrm{fm}$ (LHPC) [39], $a \simeq 0.08-0.124 \mathrm{fm}$ $(\mathrm{ETMC})[40], a \simeq 0.124 \mathrm{fm}$ (MILC) [43], and $a \simeq 0.05-0.1 \mathrm{fm}$ (CP-PACS) [42], where the intervals indicate the range of lattice spacings where the simulations were carried out.

At low quark mass, the quenched data sets show considerable differences with each other and with the unquenched sets. On the other hand, it is expected that the quenched approximation should approach the unquenched results at larger quark mass where quark loops become dynamically suppressed. This characteristic is clearly met by the PACS-CS, MILC and DeGrand results. Moreover, according to the $1 / N_{c}$ expansion, at large $N_{c}$ the plot $F_{0}$ vs $M_{\pi}$ must lie on a universal curve. This is clearly illustrated in Fig. 2, where the results for $N_{c}=3,5$ and 7 fall on an approximately common curve. This has also been shown in other LQCD calculations at varying $N_{c}[9,14]$. 
In order to properly carry out a comparison between results from different collaborations, in the analysis one could use the physical energy scale determined from the lattice and transform everything to physical units. However, this may introduce dependencies on lattice artifacts, which seem to be evident from Fig. 2 for the different unquenched calculations. In order to avoid this issue of scale matching between calculations, it is convenient to compare dimensionless ratios of observables. As mentioned earlier, $\stackrel{\circ}{F}_{0}\left(N_{c}\right)$ will be used to define these dimensionless ratios. Recently, the FLAG collaboration [45] has carried out a detailed study providing average values, namely:

$$
\begin{aligned}
\stackrel{\circ}{F}_{\pi}(\mathrm{FLAG}) & =86 \pm 1 \mathrm{MeV}, \\
\bar{l}_{4}(\mathrm{FLAG}) & =4.4 \pm 0.4
\end{aligned}
$$

In the present work only a subset of lattice data sets is used. In order to check consistency with the world averages, an analysis of those sets is performed. In the unquenched case, for $N_{c}=3, \stackrel{\circ}{F}_{0}$ is obtained from lattice results using the second order results in ChPT [44],

$$
\begin{aligned}
M_{\pi}^{2} & =\stackrel{\circ}{M}_{\pi}^{2}\left(1+\frac{\stackrel{\circ}{M}_{\pi}^{2}}{32 \pi^{2} \stackrel{\circ}{F}_{\pi}}\left(\log \frac{\stackrel{\circ}{\pi}_{\pi}^{2}}{\mu^{2}}+64 \pi^{2} l_{3}(\mu)\right)\right), \\
F_{\pi} & =\stackrel{\circ}{F}_{\pi}\left(1-\frac{\stackrel{\circ}{M}_{\pi}^{2}}{16 \pi^{2} \stackrel{\circ}{F}_{\pi}}\left(\log \frac{\stackrel{\circ}{\pi}_{\pi}^{2}}{\mu^{2}}-64 \pi^{2} l_{4}(\mu)\right)\right),
\end{aligned}
$$

where $\mu$ is the renormalization scale and $\stackrel{\circ}{M}_{\pi}$ and $\stackrel{\circ}{F}_{\pi}$ are the lowest order values of these quantities; in particular $\stackrel{\circ}{M}_{\pi}^{2}=2 B m_{q}$. The LECs are given by

$$
\begin{aligned}
& l_{3}(\mu)=-\frac{1}{64 \pi^{2}}\left(\overline{l_{3}}+\log \left((138 \mathrm{MeV})^{2} / \mu[\mathrm{MeV}]^{2}\right)\right), \\
& l_{4}(\mu)=\frac{1}{64 \pi^{2}}\left(\overline{l_{4}}+\log \left((138 \mathrm{MeV})^{2} / \mu[\mathrm{MeV}]^{2}\right)\right)
\end{aligned}
$$

where from phenomenology and recent LQCD calculations the values of the LECs $\bar{l}_{3}=3.16$ and $\bar{l}_{4}=4.03$ are obtained.

To the order of accuracy needed, $\dot{M}_{\pi}$ can be replaced by $M_{\pi}$ in Eq. (12). In addition, the ratio $\stackrel{\circ}{M}_{\pi} / M_{\pi}$ remains close to unity for the whole range of pion masses. The results of the fits to unquenched LQCD are shown in Table VIII.

The results in Table VIII are quite compatible with the FLAG ones. With that agreement it is reasonable to choose a common $\stackrel{\circ}{F}_{\pi}$ given in Eq. (9) for all the unquenched LQCD results in what follows. 
TABLE VIII: Chiral limit extrapolated $\stackrel{\circ}{F}_{\pi}$ from the unquenched LQCD results used in the fits. The quoted lattice spacings from the different collaborations are used to convert to physical units. The $\mathcal{O}\left(p^{4}\right)$ formula for $F_{\pi}$, Eq. (12), is used to obtain $\stackrel{\circ}{F}_{\pi}$ and $\overline{l_{4}}$, replacing $\stackrel{\circ}{\pi}_{\pi}$ by $M_{\pi}$ as given by the lattice results.

\begin{tabular}{c|c|c|c}
\hline \hline LQCD Collaboration & $\chi_{D O F}^{2}$ & $\stackrel{\circ}{F}_{\pi}[\mathrm{MeV}]$ & $\overline{l_{4}}$ \\
\hline PACS-CS [38] & 1.49 & $88.3 \pm 1.2$ & $4.4 \pm 0.1$ \\
LHPC [39] & 0.40 & $88.4 \pm 0.5$ & $4.09 \pm 0.03$ \\
ETMC [40] & 1.46 & $88.9 \pm 1.2$ & $3.50 \pm 0.16$ \\
MILC [43] & 0.48 & $86.5 \pm 0.3$ & $4.45 \pm 0.02$ \\
\hline \hline
\end{tabular}

Now we turn to the quenched data set. Recall that we need a value for $\stackrel{\circ}{F}_{0}$ to scale our baryon masses. The difference between the quenched data sets, plus the curvature of the $N_{c}=3$ data of Ref. [12] make us suspicious of the quality of the quenched data for $F_{\pi}$ at small quark mass. We adopt a more phenomenological approach to obtain $\stackrel{\circ}{F_{0}}$ : It is obvious that quenching effects become more important at small pion masses. However, the differences between quenched and unquenched results should become smaller as the pion mass increases. This effect is noticed in comparing the quenched with the PACS-CS and MILC results in the left panel of Fig. 2. It is then reasonable to give preference to the large pion mass results of [12] at varying $N_{c}$, where quenched and full LQCD are more alike, to guide the extraction of $\stackrel{\circ}{F}_{\pi}$ in this case. The striking linear behavior suggest to use a linear function in $M_{\pi}$ and disregard the lowest pion masses, obtaining,

$$
\stackrel{\circ}{F}_{0}(\text { Ruler })=94(5) \mathrm{MeV} \text {. }
$$

The error stems from the differences observed in the extrapolated value when changing $N_{c}$ from 3 to 7 . This value is not very different from the unquenched value in Eq. (9), and in fact gives a remarkably good agreement in the ratios of baryon masses and HF splittings obtained by the different collaborations.

The right panel of Fig. 2 depicts all LQCD results for $F_{0} / \stackrel{\circ}{F}_{0}$ vs $M_{\pi} / \stackrel{\circ}{F}_{0}$, where for the unquenched results $\stackrel{\circ}{F}_{0}$ is taken as the FLAG value Eq. (9), and the ruler value Eq. (15) for the quenched results. Quenching effects and/or lattice artifacts for the ratios remain small 
for the whole range of pion masses.

The quenching effects also become unnoticeably small for the baryon mass ratios $m_{B} / \stackrel{\circ}{F}_{0}$ and $\Delta m_{B} / \stackrel{\circ}{F}_{0}$ (where $\Delta m_{B}$ indicates a HF splitting) as shown in Fig. 3. Notice that the HF splittings for $N_{c}=3$ obtained by the different LQCD collaborations give different results, although they are consistent thanks to the generous errors. Thus, because of the current dispersion in LQCD results with dynamical fermions, at present there are no significant differences between quenched and unquenched results for the discussed ratios relevant to the study carried out here.
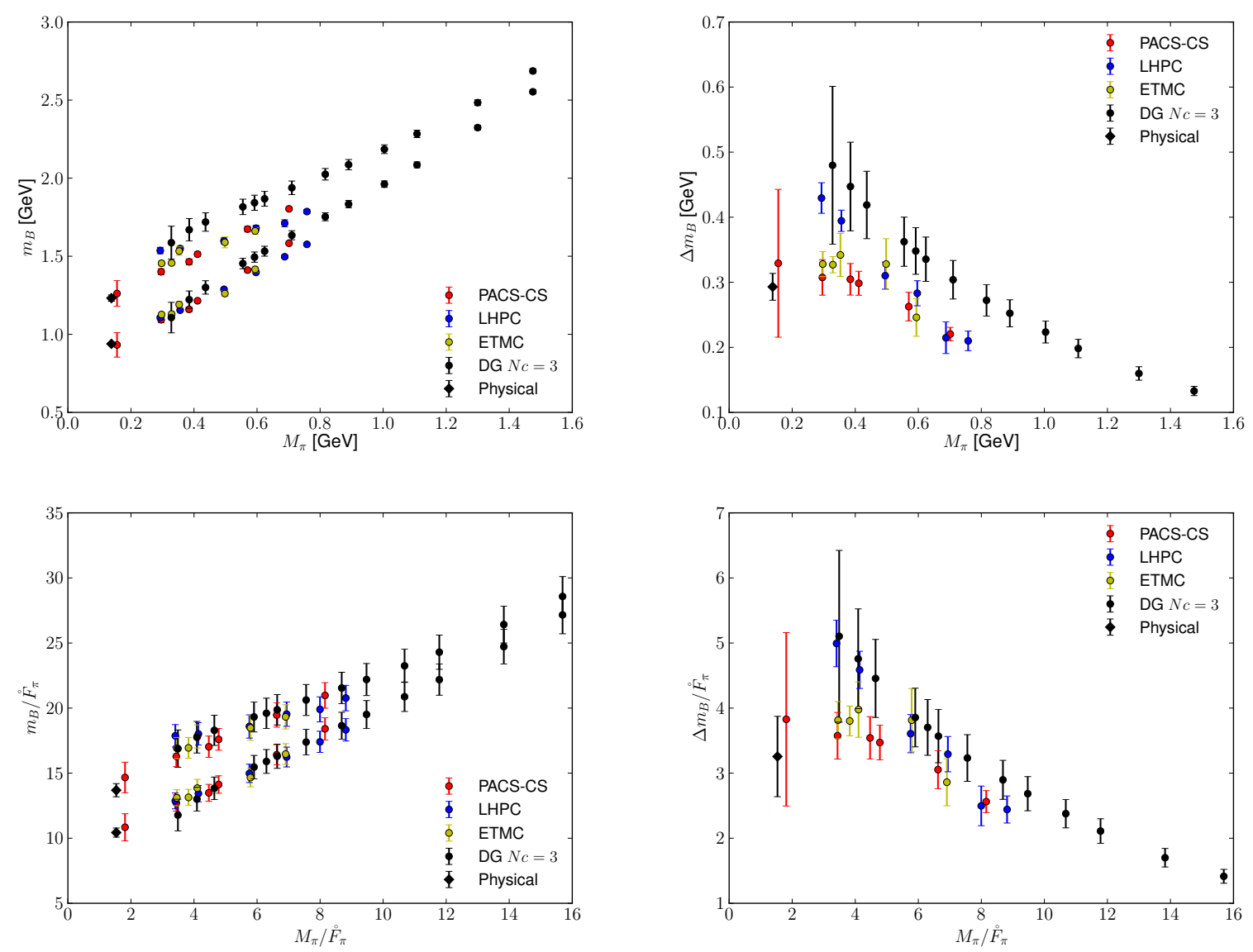

FIG. 3: Comparison for $N_{c}=3$ masses and HF splitting as functions of the pion mass of the quenched [12] and unquenched results from different collaborations in physical units and units of $\stackrel{\circ}{F}_{\pi}$. Upper panels: $m_{N, \Delta}$ (left) and $m_{\Delta}-m_{N}$ (right) vs $M_{\pi}$ in GeV. Lower panels: $m_{N, \Delta} / \stackrel{\circ}{F}_{\pi}$ (left) and $\left(m_{\Delta}-m_{N}\right) / \stackrel{\circ}{F}_{\pi}$ (right) vs $M_{\pi} / \stackrel{\circ}{F}_{\pi}$. 
In summary, although the effects of quenching can be significant for individual quantities, the ratios shown in Fig. 3 involving the baryon masses are very close for the quenched and full QCD cases, justifying the approach of combining quenched and unquenched results.

The next step is to carry out the EFT analysis of the baryon mass ratio $m_{B} / \stackrel{\circ}{F}_{0}$ vs $M_{\pi} / \stackrel{\circ}{F}_{0}$ supplemented by the HF splittings $\Delta m_{B}\left(S, S^{\prime}\right) / \stackrel{\circ}{F}_{0}$ vs $M_{\pi} / \stackrel{\circ}{F}_{0}$. The results are presented in Table IX, and can be briefly summarized as follows:

- Fit I: is a fit to $\mathcal{O}\left(\xi^{3}\right)$ in the expansion, where only the HF splittings from PACSCS [38] and DeGrand's quenched results in Sec. III are included.

- Fit II: is a combined fit where masses and HF splittings are both included. Results at different orders in the $\xi$-expansion are shown.

- Fit III: is a $\mathcal{O}\left(\xi^{3}\right)$ combined fit to masses and HF splittings but setting $\stackrel{\circ}{g}_{A}=0$. The purpose of this fit is to give a measure of the one-loop contributions in Fits I and II.

The determination of the LECs in the fits is made as follows: initially a fit of the baryon HF splittings is performed, where only the spin-dependent terms in the mass formula are needed. This is followed by a combined fit including the masses, which allows for the determination of the rest of the LECs. It should be emphasized that with the present inputs not all LECs can be determined, as discussed below.

One issue in the analysis is the value to be used for the axial coupling $\stackrel{\circ}{g}_{A}$, which determines the size of the one-loop contributions. Because of the lack of results for the axial coupling at varying $N_{c}$, it has been determined by a previous analysis from LQCD unquenched calculations at $N_{c}=3$ in Ref. [18, 20], where a combined fit to LQCD masses and the axial coupling was performed. The value obtained $\stackrel{\circ}{g}_{A}=1.4$ will be the one used here for all $N_{c}$. This corresponds to neglecting corrections $\mathcal{O}\left(1 / N_{c}\right)$ to $\stackrel{\circ}{g}_{A}$. Quenching effects also produce effects of that size on $\stackrel{\circ}{g}_{A}$. All this amounts to neglecting some $\mathcal{O}\left(\xi^{3}\right)$ effects. Determining these (expected to be small) effects will require LQCD calculations of $g_{A}$ at different values of $N_{c}$.

As emphasized in Sec. II, the HF splittings have a better behaved low energy expansion than the masses themselves. In fact they can be fitted up to $M_{\pi} \lesssim 700 \mathrm{MeV}$ with natural magnitudes for the LECs. The result is shown in Table IX as Fit I and in Fig. 4 as the dashed lines. The LQCD results show a significant $M_{\pi}$ dependency of the HF splittings, 
implying that the corrections $\mathcal{O}\left(M_{\pi}^{2} / N_{c}\right)$, determined by the loop contributions and the CT proportional to $\mu_{2}$, are important. One finds that the minimum set of LECs needed to obtain a good fit to the HF splittings reduces to $C_{H F}, C_{H F 1}, \mu_{2}$ and $z_{1}$. As shown by Eqs. (5) and (6), $C_{H F 1}$ is the LEC of an $O\left(1 / N_{c}^{2}\right)$ CT, while $\mu_{2}$ and $z_{1}$ are LECs of $\mathcal{O}\left(M_{\pi}^{2}\right)$ CTs. In the fit one finds that the LECs $\mu_{2}$ and $z_{1}$ are highly correlated and thus cannot be determined using the HF fit only. Setting one of them to vanish does not affect the $\chi^{2}$. In Fit I in Table IX $z_{1}$ was set to vanish.

The LEC $C_{H F 1}$ gives the $1 / N_{c}$ correction to the LO splitting determined by $C_{H F}$, and therefore some correlation between them is expected, as it is indeed the case: $C_{H F}$ changes significantly from the $\mathcal{O}(\xi)$ fit to the $\mathcal{O}\left(\xi^{3}\right)$ fit. The LEC $z_{1}$ along with the rest of the LECs are most sensitive to the baryon masses, and are determined in the combined fit. A detailed analysis of the fits shows that the LECs $m_{2}, m_{3}, w_{1}, w_{2}, C_{H F 2}$ and $\mu_{1}$ have marginal effects (due to correlations with the rest of the LECs), and they are therefore be set to vanish.

After the fit to the HF splittings, the combined fit including baryon masses is carried out. The results are shown in Table IX as Fit II and in Figs. 4 and 5 as the solid lines. The errors shown in the Table represent the $68 \%$ confidence interval and the bands are the corresponding Monte Carlo propagation of the LECs' errors. The values of the LECs $C_{H F}$ and $C_{H F 1}$ previously determined by the HF fit to $\mathcal{O}\left(\xi^{3}\right)$ change within errors, and the correlation between $\mu_{2}$ and $z_{1}$ is now eliminated and both LECs can be determined. The new result for the HF splittings is consistent with the ones of the Fit I as shown in Fig. 4, where the dashed lines of Fit I and full lines of Fit II fall inside the error bands. The LEC $z_{1}$ is needed in order to obtain a good description of the masses. This parameter is the leading in $1 / N_{c}$ term contributing to the wave function renormalization and the value obtained here perfectly agrees with the one obtained for $N_{c}=3$ in Ref. [18], where also the axial couplings were simultaneously analyzed.

In contrast to the HF splittings, the spin-flavor singlet component is naturally big, $\mathcal{O}\left(N_{c}\right)$, and receives corrections of the same order in $N_{c}$ but higher order in $M_{\pi}$. Thus, the convergence of the low-energy expansion is poorer, with the situation increasingly worsening as $N_{c}$ increases. In contrast to the HF splittings, the combined fit only has a range of validity at most up to $M_{\pi} \sim 600 \mathrm{MeV}$. For this reason the fits only include results with $M_{\pi}<600 \mathrm{MeV}$. The LEC $m_{1}$ represents a $1 / N_{c}$ correction to the term proportional to $m_{0}$, and although there is some correlation among them, $m_{1}$ is very important. This is shown by Fig. 5, which 
TABLE IX: LECs from fits to HF splittings (Fit I) and masses (Fits II and III) at varying $N_{c}$ and a given order in the $\xi$-expansion $\mathcal{O}\left(\xi^{\nu}\right)$. In the fits only data up to $M_{\pi} \sim 600 \mathrm{MeV}$ are included. In Fits I and II, $\stackrel{\circ}{g}_{A}=1.4$ and the renormalization scale is set at $\mu=740 \mathrm{MeV}$. In Fit III, $\stackrel{\circ}{g}_{A}=0$. The LECs are given in units of $\stackrel{\circ}{F}_{0}$ in the first set of rows. The second set of rows show the estimated values in physical units using $\stackrel{\circ}{F}_{\pi}=90 \mathrm{MeV}$.

\begin{tabular}{|c|c|c|c|c|c|c|c|c|c|}
\hline \multicolumn{10}{|c|}{ LECs in units of $\stackrel{\circ}{F}_{0}$} \\
\hline Fit & Order & $\chi_{\mathrm{DOF}}^{2}$ & $m_{0}$ & $m_{1}$ & $C_{H F}$ & $c_{1}$ & $C_{H F 1}$ & $\mu_{2}$ & $z_{1}$ \\
\hline I & 3 & 0.70 & 0 & 0 & $0.39(9)$ & 0 & $6.7(3)$ & $-0.040(4)$ & 0 \\
\hline \multirow{3}{*}{ II } & 1 & 1.48 & $6.0(2)$ & $-8.6(7)$ & $2.86(6)$ & $0.052(2)$ & 0 & 0 & 0 \\
\hline & 2 & 1.07 & $5.7(2)$ & $-8.4(6)$ & $0.96(9)$ & $0.149(3)$ & $6.3(6)$ & 0 & 0 \\
\hline & 3 & 0.89 & $4.0(2)$ & $-3.8(7)$ & $0.61(9)$ & $0.266(5)$ & $6.1(4)$ & $0.017(3)$ & $-0.0080(4)$ \\
\hline III & 3 & 0.73 & $6.2(2)$ & $-9.2(6)$ & $2.7(3)$ & $0.050(2)$ & $4.2(7)$ & $-0.004(2)$ & $-0.0040(5)$ \\
\hline \multicolumn{10}{|c|}{ LECs in physical units } \\
\hline Fit & Order & $\chi_{\mathrm{DOF}}^{2}$ & $m_{0}[\mathrm{MeV}]$ & $m_{1}[\mathrm{MeV}]$ & $C_{H F}[\mathrm{MeV}]$ & $c_{1}\left(10^{-3}\right)\left[\mathrm{MeV}^{-1}\right]$ & $C_{H F 1}[\mathrm{MeV}$ & $\mu_{2}\left(10^{-3}\right)\left[\mathrm{MeV}^{-1}\right]$ & $z_{1}\left(10^{-6}\right)\left[\mathrm{MeV}^{-2}\right]$ \\
\hline I & 3 & 0.70 & 0 & 0 & $35(8)$ & 0 & $607(31)$ & $-0.42(4)$ & 0 \\
\hline \multirow{3}{*}{ II } & 1 & 1.48 & $543(20)$ & $-775(60)$ & $257(6)$ & $0.58(3)$ & 0 & 0 & 0 \\
\hline & 2 & 1.07 & $515(20)$ & $-752(60)$ & $86(8)$ & $1.66(3)$ & $566(60)$ & 0 & 0 \\
\hline & 3 & 0.89 & $365(20)$ & $-345(60)$ & $52(7)$ & $2.96(5)$ & $546(40)$ & $0.20(4)$ & $-0.97(4)$ \\
\hline III & 3 & 0.73 & $553(20)$ & $-827(50)$ & $242(22)$ & $0.50(2)$ & $375(60)$ & $-0.04(2)$ & $-0.45(6)$ \\
\hline \multicolumn{3}{|c|}{ Natural value } & $\sim 300$ & $\sim$ & $\sim 300$ & $\sim 10^{-3}$ & $\sim$ & $\sim 10^{-3}$ & $\sim 10^{-6}$ \\
\hline
\end{tabular}

gives some evidence that the spin-flavor singlet part of the masses deviates from the simple linear behavior in $N_{c}$.

Evidently it will take a more extensive set of inputs at varying $N_{c}$ to fix all the LECs, with more results in the lower range of $M_{\pi}$ and possibly also larger values of $N_{c}$ than presently available. This obviously represents a difficult challenge at this time. In order to study the stability of the low-energy expansion, combined fits were performed at different orders in $\xi$. The pattern of convergence of the spin-singlet LECs is stable. The pion mass dependence of the HF splitting only appears at $\mathcal{O}\left(\xi^{3}\right)$, through $\mu_{2}$, and therefore at lower orders one can only obtain a rough description. Clearly, $C_{H F}$ is very sensitive to the order of the expansion, because it is strongly correlated with $C_{H F 1}$.

A comparison with the results for LECs obtained for $N_{c}=3$ in Ref. [18] requires the 
following identifications, where on the left is the result of that fit and on the right the result of the present analysis: $m_{0} \rightarrow m_{0}+\frac{1}{3} m_{1}, C_{H F} \rightarrow C_{H F}+\frac{1}{3} C_{H F 1}, c_{1} \rightarrow c_{1}+\frac{1}{3} \mu_{1}, \mu_{2} \rightarrow \mu_{2}$ and $z_{1} \rightarrow z_{1}$. The respective comparisons are as follows: 250(30) MeV vs 310(15) MeV, 234(15) $\mathrm{MeV}$ vs $300(36) \mathrm{MeV}, 0.00296(5)$ vs $0.00235(4)$ ( $\mu_{1}$ set to zero), $0.20(4) \times 10^{-3} \mathrm{MeV}^{-1}$ vs $-7.3(6) \times 10^{-3} \mathrm{MeV}^{-1}$, and $-9.7(4) \times 10^{-7} \mathrm{MeV}^{-2}$ vs $-8.9(2) \times 10^{-7} \mathrm{MeV}^{-2}$. These results are in reasonable agreement, taking into account that the LECs obtained in Ref. [18] were obtained from combined fits to masses and the axial charges. Only $\mu_{2}$ is clearly in disagreement. Below the origin of the instability in the determination of $\mu_{2}$ will be discussed.

An additional test of consistency between quenched and unquenched results is carried out by performing separate fits at $N_{c}=3$ with only quenched or only unquenched LQCD results. The results of those fits are shown in Table X: the first fit only includes unquenched PACS-CS data, the second only quenched DeGrand data, and finally a third fit including both PACS-CS and DeGrand data. Results are shown in physical units using $\stackrel{\circ}{*}_{\pi}=90$ $\mathrm{MeV}$ as the unit to transform. Also shown are the extrapolated nucleon and $\Delta$ masses to the physical point. The independent fits in Table $\mathrm{X}$ show compatibility of the quenched

TABLE X: Independent fits to mass and HF splitting ratios of $N_{c}=3$ LQCD data sets. To convert to physical units we use ${\stackrel{\circ}{F_{\pi}}}=90 \mathrm{MeV}$

\begin{tabular}{c|c|c|c|c|c|c|c|c}
\hline \hline LQCD Collaboration & $\chi_{\text {DOF }}^{2}$ & $m_{0}[\mathrm{MeV}]$ & $C_{H F}[\mathrm{MeV}]$ & $c_{1}\left(10^{-3}\right)\left[\mathrm{MeV}^{-1}\right]$ & $\mu_{2}\left(10^{-3}\right)\left[\mathrm{MeV}^{-1}\right]$ & $z_{1}\left(10^{-6}\right)\left[\mathrm{MeV}^{-2}\right]$ & $m_{N}[\mathrm{MeV}]$ & $m_{\Delta}[\mathrm{MeV}]$ \\
\hline PACS-CS & 0.47 & $253(3)$ & $176(8)$ & $2.5(1)$ & $-0.3(1)$ & $-0.7(1)$ & $941(2)$ & $1190(10)$ \\
\hline DeGrand & 0.46 & $249(4)$ & $184(9)$ & $2.6(2)$ & $-0.09(8)$ & $-0.7(1)$ & $939(1)$ & $1203(12)$ \\
\hline PACS-CS + DeGrand & 0.67 & $255(3)$ & $166(7)$ & $2.6(1)$ & $-0.06(5)$ & $-0.76(8)$ & $942(2)$ & $1178(8)$ \\
\hline \hline
\end{tabular}

and unquenched results at $N_{c}=3$. This lends a strong support to the approach used here for combining results at the level of masses in units of $\stackrel{\circ}{F}_{\pi}$. One therefore concludes that, given that quenched and full QCD are formally different at sub leading order in $1 / N_{c}$, the phenomenological approach followed here shows that such differences are not clearly noticeably in the present analysis.

It is important to test the one loop contributions in the description of the LQCD results. In order to expose them, a combined fit is carried out at $\mathcal{O}\left(\xi^{3}\right)$ in which the coupling $\stackrel{\circ}{g}_{A}$ is 

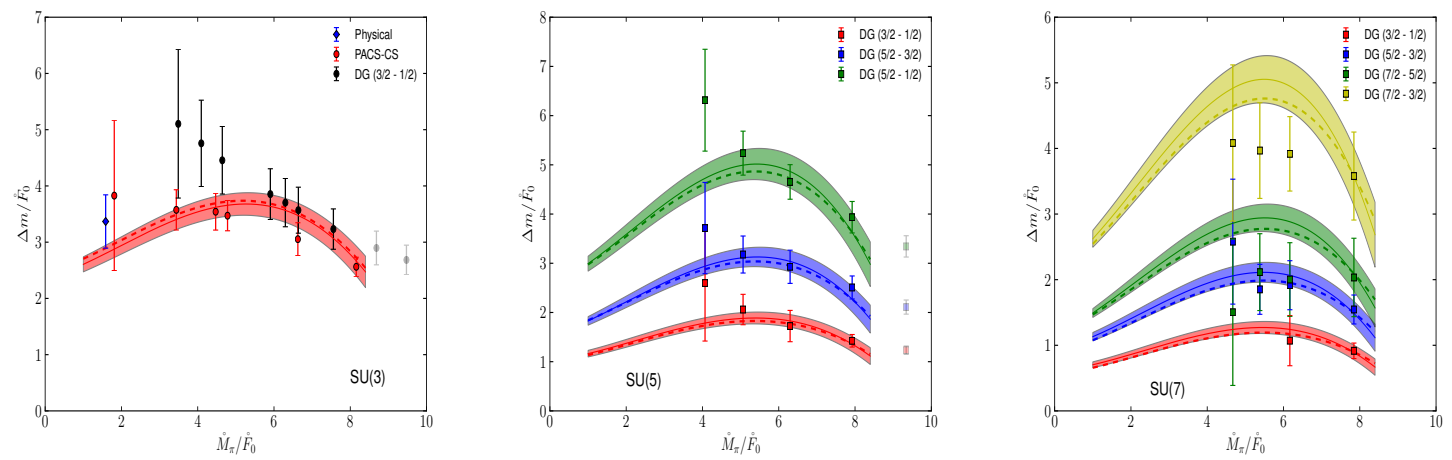

FIG. 4: HF splittings in the $N_{c}=3,5$ and 7 multiplets. Results are in units of $\stackrel{\circ}{F}_{0} . N_{c}=3$ includes the results from PACS-CS [38], and the shaded points represent lattice points excluded in the fit, which correspond to pion masses $M_{\pi} \gtrsim 800 \mathrm{MeV}$. Dashed lines for fits when only HF lattice results are included, and the solid lines for fits when masses are also included.
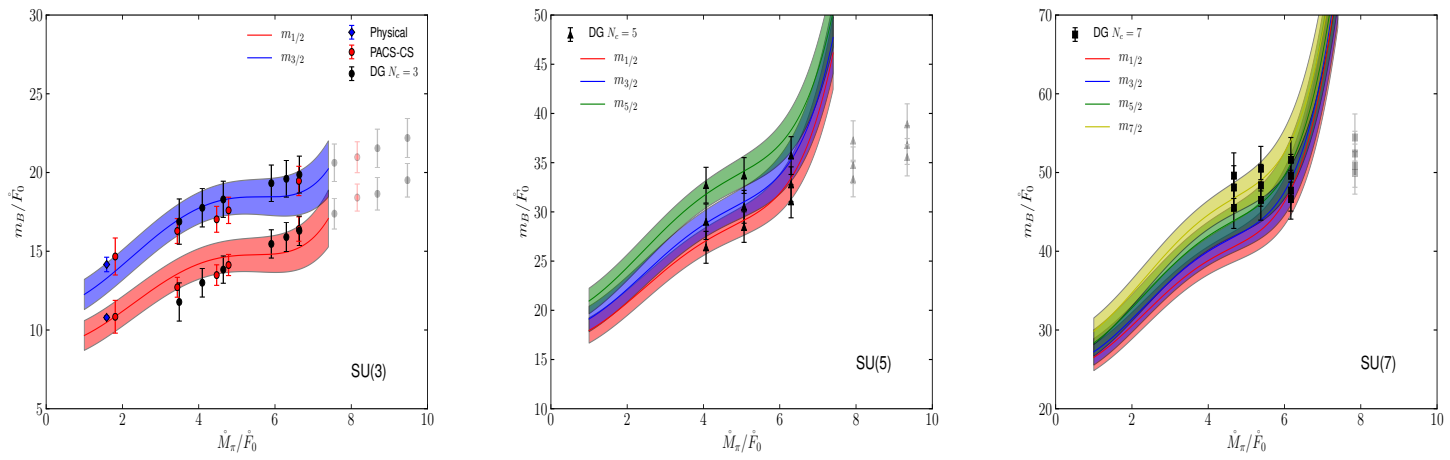

FIG. 5: Baryon masses for $N_{c}=3,5$ and 7. Results in units of $\stackrel{\circ}{F}_{0}=\stackrel{\circ}{F_{\pi}} \sqrt{3 / N_{c}} \cdot N_{c}=3$ includes the results from PACS-CS [38]. Shaded points represent lattice points excluded in the fit which have an approximated pion mass of $M_{\pi} \gtrsim 700 \mathrm{MeV}$.

set to vanish (Fit. III in Table IX and Figs. 6 and 7). As expected, the fit can be consistently carried out for larger values of $M_{\pi}$. When a similar range of $M_{\pi}$ is used, the LECs show larger error bars, which can be explained by the fact that in the case where $\stackrel{\circ}{g}_{A}=1.4$ there must be important cancellations between loop contributions and counter-terms leading to tighter error bars. The impact of the loop contributions can be seen in the very different chiral extrapolations obtained with and without the one loop contributions. 

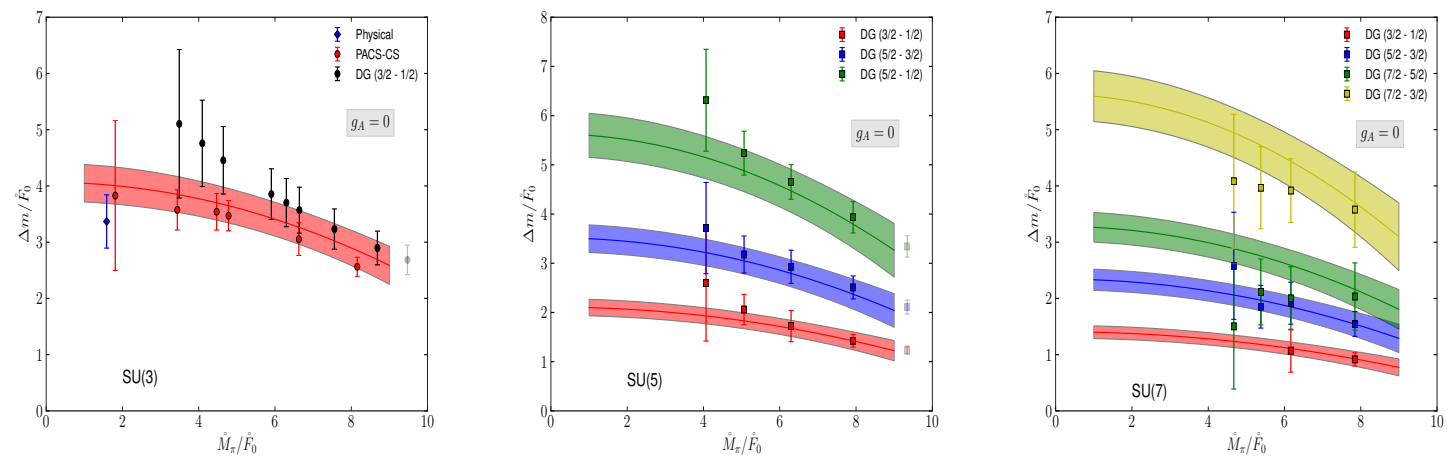

FIG. 6: HF splittings in the $N_{c}=3,5$ and 7 multiplets obtained by setting $\stackrel{\circ}{g}_{A}=0$. Results are in units of $\stackrel{\circ}{F}_{0}$. For $N_{c}=3$, the results of PACS-CS [38] are also included.
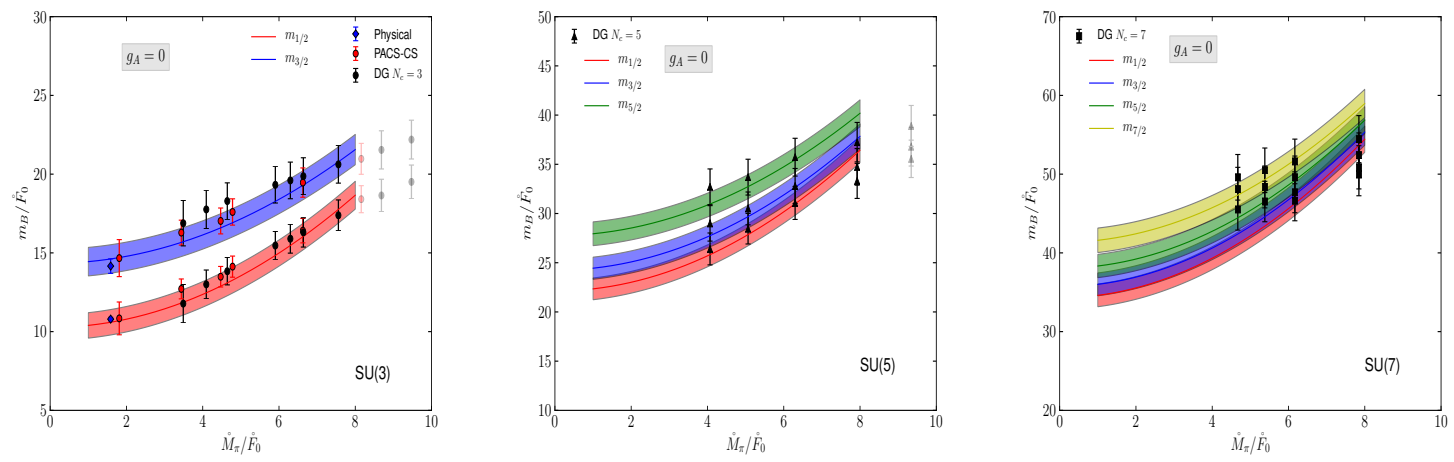

FIG. 7: Baryon masses $N_{c}=3,5$ and 7 obtained by setting $\stackrel{\circ}{g}_{A}=0$. Results are in units of $\stackrel{\circ}{F}_{0}$. For $N_{c}=3$, the results of PACS-CS [38] are also included.

Let us estimate the range in $M_{\pi}$ where the EFT seems to work. This can be estimated by taking as the upper boundary of that range the upper inflection points of the curves in Fig. 5. This gives a range approximately described by $M_{\pi} / \stackrel{\circ}{F}_{0}<6.25-0.25 N_{c}$.

Finally, the $1 / N_{c}$ expansion, where the link $1 / N_{c}=\mathcal{O}\left(p^{2}\right)$ is used, turns out to give poor fits, even when one removes the inputs at lower values of $M_{\pi}$ where it should not work. The only way it can give a reasonable fit is if the effects of the wave function renormalization are enormous. This is unrealistic.

The analysis leads to the following observations:

1. In the range of $M_{\pi}$ considered, the chiral loop contributions are very important, driving the curvature observed in the HF splittings, and giving extrapolations of the baryon 
masses to small pion mass which are very different than in the case where the loop contributions are excluded. This effect increases with $N_{c}$.

2. The fact that the range of applicability in $M_{\pi}$ of the EFT diminishes with increasing $N_{c}$, means that LQCD results for $N_{c}=5$ and 7 at smaller quark masses than the ones presented in this work would be necessary for a more significant analysis. In addition, a proper analysis requires unquenched results.

3. The $N_{c}=7$ inputs have played a lesser role in the results of the analysis because they are mostly located in a range of pion masses where the convergence of the EFT is unwarranted. Only results at lowest three values of $M_{\pi}$ could be included in the fits.

4. The naturalness of the results is reflected in the fact that in units of GeV the LECs are all of order one, as one would expect from a well behaved expansion.

5. The two new effects that have become accessible with the LQCD results are the subleading in $1 / N_{c}$ terms in the spin-flavor singlet component of the baryon masses and in the HF splittings. The LECs associated with those effects, namely $m_{1}$ and $C_{H F 1}$ respectively, have been determined. The latter one is larger than the expected natural size, but it must be noticed that it is very strongly correlated with $C_{H F}$, which is the leading $H F$ LEC.

6. The LQCD results show clearly that the HF splittings decrease with increasing quark mass in approximately the same proportion for all values of $N_{c}$. This behavior has contributions from the chiral loop as well as the LEC $\mu_{2}$. There is significant curvature as shown if Fig. 4 due to the loop contributions. This makes the determination of $\mu_{2}$ rather uncertain. In fact, its value is sensitive to the range of $M_{\pi}$ considered in the fit. It is possible that one could use the stability of $\mu_{2}$ to set the range in $M_{\pi}$ where the fit to the HF splittings can be safely done with the EFT.

\section{CONCLUSIONS}

Understanding the $N_{c}$ dependence of gauge theories is a fundamental theoretical problem, which also has profound phenomenological consequences for QCD. In fact, QCD phenomenology in both mesons and baryons indicates that an expansion in powers of $1 / N_{c}$ seems 
to work for most observables. The actual study of QCD at different values of $N_{c}$ is essential for confirming that observation. LQCD provides the only present means for those studies beyond $N_{c}=3$. These studies are essential for quantifying the sub-leading in $1 / N_{c}$ effects. In the case of light baryons studied in this work, the aim has been to use the LQCD results up to $N_{c}=7$ in order to elucidate sub-leading in $1 / N_{c}$ effects as described by a low energy EFT. The study proved to be fruitful, showing consistency with previous results obtained by analyzing the $N_{c}=3 \mathrm{LQCD}$ results, and in addition it permitted the determination of two sub-leading effects, namely the sub-leading correction to the spin-flavor singlet component of the baryon masses, which is entirely given by the LEC $m_{1}$, and the sub-leading corrections to the HF splittings which stem from loop contributions and the LEC $C_{H F 1}$. Other sub-leading effects which are in principle present could not be determined because of the still significant error bars in the LQCD results.

It is observed that the different LQCD results for the HF splittings are not entirely compatible. Since HF effects are dominated by short distance contributions, they may be very sensitive to lattice artifacts, and might require careful continuum extrapolations. It is clearly desirable to have more accurate HF results, in particular because the HF effects are better behaved in the EFT than the masses themselves.

It is clear that the problem of chiral extrapolations of baryon masses (not of HF splittings) becomes more severe as a consequence of the shrinking domain of the low energy expansion with increasing $N_{c}$. In order to improve the study of this work, it will be necessary to have $N_{c}>3 \mathrm{LQCD}$ results for smaller quark masses than the ones analyzed. For a fully consistent study the next step should include dynamical quarks, in particular because of the problem of exceptional configurations which arises in quenched QCD as the quark mass decreases. This task is clearly very challenging, but we believe that it is not impossible, merely expensive. It is one that will give important additional insights into the $1 / N_{c}$ expansion for light baryons. Finally, the extension to three flavors based on the recent results [26] can be already analyzed in the same way as the case of two flavor presented here.

\section{Acknowledgments}

T. D. thanks JLab for its hospitality, where this project was initiated. The conversion of the MILC code to arbitrary number of colors was done by T. D. with Y. Shamir 
and B. Svetitsky. This work was supported in part by the U. S. Department of Energy under grant DE-FG02-04ER41290 (T. D.), by DOE Contract No. DE-AC05-06OR23177 under which JSA operates the Thomas Jefferson National Accelerator Facility (A.C.C and J. L. G.), and by the National Science Foundation (USA) through grant PHY-0855789 and PHY-1307413 (J. L. G.). A.C.C also thanks the financial support from the EU-Research Infrastructure Integrating Activity, "Study of Strongly Interacting Matter"(HadronPhysics2, grant n.227431) under the Seventh Framework Program of the EU. 


\section{Appendix A: UV finite parts of the self-energy}

The one-loop finite contributions to the self-energy relevant for the masses at $\mathcal{O}\left(\xi^{3}\right)$ are as follows:

$$
\begin{aligned}
& \delta \Sigma_{\text {finite }}\left(\mathfrak{p}^{0}=0\right)(S)=\frac{\stackrel{\circ}{9}_{A}^{2}\left(N_{c}+4\right)}{128 \pi^{2} \stackrel{\circ}{0}_{0}^{2}} \\
& \times\left\{\frac{2\left(3(2 S-1) A_{1}(S-1)-3(2 S+3) A_{1}(S)+8 C_{H F}^{3}(2 S+1)(5 S(S+1)+3)\left(4-3 \log \left(\frac{M_{\pi}}{\mu}\right)\right)\right)}{3 N_{c}^{3}(2 S+1)}\right. \\
& +\pi\left(\frac{4 C_{H F}^{2}\left((2 S-1) S^{2} R(S-1)+(S+1)^{2}(2 S+3) R(S)\right)}{N_{c}^{3}(2 S+1)}-M_{\pi}^{3}+\frac{M_{\pi}^{2}((1-2 S) R(S-1)-(2 S+3) R(S))}{N_{c}(2 S+1)}\right) \\
& \left.+\frac{3 C_{H F} M_{\pi}^{2}\left(6 \log \left(\frac{M_{\pi}}{\mu}\right)-7\right)}{N_{c}}\right\} \\
& \delta Z_{\text {finite }}(S)=\frac{3 \stackrel{\circ}{g}_{A}^{2}\left(N_{c}+4\right)}{64 \pi^{2} \stackrel{\circ}{F}_{0}^{2}}\left\{\frac { 1 } { N _ { c } ^ { 2 } } \left(\frac{C_{H F}\left(S(2 S-1) A_{2}(S-1)+(S+1)(2 S+3) A_{2}(S)\right)}{(2 S+1)}\right.\right. \\
& \left.+2 C_{H F}^{2}(2 S(S+1)+3)\left(\log \left(\frac{M_{\pi}}{\mu}\right)-1\right)+\frac{\pi C_{H F}(S(2 S-1) R(S-1)-(S+1)(2 S+3) R(S))}{2(2 S+1)}\right) \\
& \left.+\frac{1}{8} M_{\pi}^{2}\left(3-6 \log \left(\frac{M_{\pi}}{\mu}\right)\right)\right\}
\end{aligned}
$$

where :

$$
\begin{aligned}
R(S) & =\sqrt{N_{c}^{2} M_{\pi}^{2}-4(S+1)^{2} C_{H F}^{2}} \\
A_{1}(S) & =\left(-N_{c}^{2} M_{\pi}^{2}+4(S+1)^{2} C_{H F}^{2}\right)^{\frac{3}{2}} \operatorname{arctanh}\left(\frac{2(S+1) C_{H F}}{\sqrt{-N_{c}^{2} M_{\pi}^{2}+4(S+1)^{2} C_{H F}^{2}}}\right) \\
A_{2}(S) & =\frac{A_{1}(S)}{-N_{c}^{2} M_{\pi}^{2}+4(S+1)^{2} C_{H F}^{2}}
\end{aligned}
$$

The terms in Eqns. (A1) and (A2) involving $R, A_{1}$ and $A_{2}$ are due to the contributions of the baryons with spins $S \pm 1$ in the loop. 
[1] M. Teper, Phys.Lett. B397, 223 (1997), hep-lat/9701003.

[2] B. Lucini and M. Teper, JHEP 0106, 050 (2001), hep-lat/0103027.

[3] L. Del Debbio, H. Panagopoulos, P. Rossi, and E. Vicari, JHEP 0201, 009 (2002), hepth/0111090.

[4] C. Allton, M. Teper, and A. Trivini, JHEP 0807, 021 (2008), 0803.1092.

[5] B. Lucini, M. Teper, and U. Wenger, JHEP 0406, 012 (2004), hep-lat/0404008.

[6] B. Lucini, A. Rago, and E. Rinaldi, JHEP 1008, 119 (2010), 1007.3879.

[7] L. Del Debbio, B. Lucini, A. Patella, and C. Pica, JHEP 0803, 062 (2008), 0712.3036.

[8] G. S. Bali and F. Bursa, JHEP 0809, 110 (2008), 0806.2278.

[9] G. S. Bali, F. Bursa, L. Castagnini, S. Collins, L. Del Debbio, et al., JHEP 1306, 071 (2013), 1304.4437.

[10] R. Narayanan and H. Neuberger, Phys.Lett. B616, 76 (2005), hep-lat/0503033.

[11] A. Hietanen, R. Narayanan, R. Patel, and C. Prays, Phys.Lett. B674, 80 (2009), 0901.3752.

[12] T. DeGrand, Phys.Rev. D86, 034508 (2012), 1205.0235.

[13] E. Vicari and H. Panagopoulos, Phys.Rept. 470, 93 (2009), 0803.1593.

[14] B. Lucini and M. Panero, Phys.Rept. 526, 93 (2013), 1210.4997.

[15] E. E. Jenkins, Phys.Rev. D53, 2625 (1996), hep-ph/9509433.

[16] R. Flores-Mendieta, E. E. Jenkins, and A. V. Manohar, Phys.Rev. D58, 094028 (1998), hep$\mathrm{ph} / 9805416$.

[17] R. Flores-Mendieta and C. P. Hofmann, Phys.Rev. D74, 094001 (2006), hep-ph/0609120.

[18] A. Calle Cordon and J. L. Goity, Phys.Rev. D87, 016019 (2013), 1210.2364.

[19] A. Calle Cordon and J. L. Goity, PoS QNP2012, 136 (2012), 1209.0030.

[20] A. Calle Cordon and J. L. Goity (2013), 1303.2126.

[21] E. E. Jenkins and A. V. Manohar, Phys.Lett. B255, 558 (1991).

[22] E. E. Jenkins and A. V. Manohar, Phys.Lett. B259, 353 (1991).

[23] R. F. Dashen and A. V. Manohar, Phys.Lett. B315, 438 (1993), hep-ph/9307242.

[24] R. F. Dashen and A. V. Manohar, Phys.Lett. B315, 425 (1993), hep-ph/9307241.

[25] R. Flores-Mendieta, M. A. Hernandez-Ruiz, and C. P. Hofmann, Phys.Rev. D86, 094041 (2012), 1210.8445 . 
[26] T. DeGrand, Phys.Rev. D89, 014506 (2014), 1308.4114.

[27] J.-L. Gervais and B. Sakita, Phys.Rev.Lett. 52, 87 (1984).

[28] J.-L. Gervais and B. Sakita, Phys.Rev. D30, 1795 (1984).

[29] T. D. Cohen, Rev.Mod.Phys. 68, 599 (1996).

[30] R. Kaiser and H. Leutwyler, Eur.Phys.J. C17, 623 (2000), hep-ph/0007101.

[31] J. N. Labrenz and S. R. Sharpe, Phys.Rev. D54, 4595 (1996), hep-lat/9605034.

[32] S. R. Sharpe, Phys.Rev. D56, 7052 (1997), hep-lat/9707018.

[33] C.-K. Chow and S.-J. Rey (1997), hep-ph/9712528.

[34] A. Hasenfratz, R. Hoffmann, and S. Schaefer, JHEP 0705, 029 (2007), hep-lat/0702028.

[35] URL http://www.physics.utah.edu/ detar/milc/.

[36] R. Sommer, Nucl.Phys. B411, 839 (1994), hep-lat/9310022.

[37] A. Bazavov, D. Toussaint, C. Bernard, J. Laiho, C. DeTar, et al., Rev.Mod.Phys. 82, 1349 (2010), 0903.3598.

[38] S. Aoki et al. (PACS-CS Collaboration), Phys.Rev. D79, 034503 (2009), 0807.1661.

[39] A. Walker-Loud, H.-W. Lin, D. Richards, R. Edwards, M. Engelhardt, et al., Phys.Rev. D79, 054502 (2009), 0806.4549.

[40] C. Alexandrou, E. B. Gregory, T. Korzec, G. Koutsou, J. W. Negele, et al., Phys.Rev.Lett. 107, 141601 (2011), 1106.6000.

[41] E. Witten, Nucl.Phys. B156, 269 (1979).

[42] S. Aoki et al. (CP-PACS Collaboration), Phys.Rev. D67, 034503 (2003), hep-lat/0206009.

[43] C. Aubin et al. (MILC Collaboration), Phys.Rev. D70, 114501 (2004), hep-lat/0407028.

[44] J. Gasser and H. Leutwyler, Annals Phys. 158, 142 (1984).

[45] S. Aoki, Y. Aoki, C. Bernard, T. Blum, G. Colangelo, et al. (2013), 1310.8555. 